\title{
Effets comparés de l'aspersion mécanique de l'urée en solution sur andain au champ et manuelle sur bottes pour le traitement de la paille de blé sur sa digestibilité et sur la croissance d'agneaux
}

\author{
Mohamed Houmani* \\ Laboratoire de digestibilité animale, institut d'agronomie, université de Blida, \\ 09100 Blida, Algérie
}

(Reçu le 21 octobre 1997 ; accepté le 20 février 1998)

\begin{abstract}
Comparison of mechanically spraying urea in solution in the field or manually spraying the sheaves for treating wheat straw. Effects on digestibility and growth of lambs. Treating straw with a urea solution can increase its nitrogen level from 3.3 to $7.1 \%$ when the treatment method used is mechanical spraying in the field (PTUA) and from 3.3 to $10.8 \%$ when the sheaves are manually sprayed (PTUB). The increase in the amounts ingested was $34 \%$ for the PTUA straw and $39 \%$ for the PTUB straw in adult sheep. In growing lambs, the increase was only $18.2 \%$ and $19.8 \%$ for the PTUA and PTUB straws, respectively. The difference between the quantities ingested by the adult and the growing animals can be explained by the fact that the lambs were fed a different amount of complement. The two spraying techniques used improved the digestibility of the treated straw by the same proportion with respect to untreated straw (PNT). The increase in growth attained by the lambs was slightly greater with the PTUA straw (112 g/day) as compared to the PTUB straw (103 g/day) or PNT straw (104 g/day). However, both PTUA and PTUB diets involved the same amount of complement, whereas the PNT diet involved more. Therefore, the PTUA and PTUB diets were $8-12 \%$ cheaper per kg of growth. (@) Elsevier/Inra)
\end{abstract}

straw / mechanical and manual spraying / urea / digestibility / lambs / growth / feeding cost

Résumé - Le traitement à l'urée en solution fait passer la teneur en matières azotées totales de la paille de 3,3 à 7,1\% avec l'aspersion mécanique sur andain au champ (PTUA) et de 3,3 à $10,8 \%$ avec l'aspersion manuelle sur bottes (PTUB). L'amélioration des quantités ingérées est de $34 \%$ avec la paille PTUA et $39 \%$ avec la paille PTUB pour des moutons adultes ; chez des agneaux en croissance, cette amélioration des quantités ingérées n'est que de $18,1 \%$ avec la paille PTUA et de 19,8\% avec la paille PTUB. Cette différence serait due à la quantité différente

\footnotetext{
* Correspondance et tirés à parts

Fax : (213) 3.43.36.39;3.43.38.64; 3.43.38.63.
} 
des compléments distribués aux moutons adultes et aux agneaux en croissance. Les deux techniques d'aspersion utilisées améliorent la digestibilité de la paille traitée dans les mêmes proportions par rapport à la paille non traitée. Le croît réalisé par les agneaux est un peu plus élevé avec le régime contenant de la paille PTUA $(112 \mathrm{~g} / \mathrm{j})$ qu'avec les régimes contenant de la paille PTUB $(103 \mathrm{~g} / \mathrm{j})$ à même niveau de complémentation ou contenant de la paille PNT $(104 \mathrm{~g} / \mathrm{j})$ mais avec une complémentation plus importante. Les régimes contenant de la paille PTUA et PTUB permettent une économie alimentaire de 4 à 6 Dinars algériens par kg de croît. (C Elsevier/Inra)

paille / traitement / urée / digestibilité / ingestibilité / agneaux / croissance / coût alimentaire

\section{INTRODUCTION}

L'utilisation de l'urée comme source d'ammoniac pour le traitement de la paille est connue depuis plusieurs années $[5,15$, $18,27]$. La technique de traitement repose sur l'aspersion manuelle d'une solution d'urée de l'ordre de 30 à $50 \%$ du poids de la paille, ce qui la rend pénible et coûteuse en main d'œuvre surtout lorsque les quantités de paille à traiter sont importantes. C'est pour ces raisons que le traitement des pailles de céréales à l'urée a finalement été assez peu pratiqué dans certains pays comme l'Algérie où le déficit fourrager dépasse 4,4 milliards d'UFL et, où la production annuelle de pailles peut atteindre 3,5 millions de tonnes [20].

En régions tempérées, Chenost et Besle [9], pour faciliter le traitement à l'urée, ont envisagé la mécanisation de l'opération au champ au moment de la récolte suivie d'un conditionnement de la paille en balles.

En Afrique du Nord, la récolte des céréales a lieu en été par climat chaud et sec ; malgré de nombreux travaux entrepris sur la valorisation des pailles par traitement à l'urée par différents auteurs dont Chermiti et al. [10], Chermiti et al. [11], Guessous et Rihani [16], Lawrence et al. [23], aucun n'a proposé de solution à cette contrainte physique et matérielle que constitue l'aspersion manuelle. C'est pour cela que nous avons envisagé de mécaniser l'opération en aspergeant la paille en andain au champ juste avant de la presser et de la récolter en bottes rectangulaires.
L'objet de cette étude est de rapporter les résultats de deux essais entrepris dans le but : 1) de tester la possibilité de mécaniser l'opération de traitement de la paille à l'urée directement sur andain au champ au moment de la récolte dans les conditions climatiques des hautes plaines céréalières algériennes; 2) d'évaluer, par comparaison au traitement manuel sur bottes, l'intérêt pour des agneaux en croissance.

\section{MATÉRIEL ET MÉTHODES}

\subsection{Traitement de la paille à l'urée sur andain au champ (paille PTUA)}

La paille a été aspergée sur andain avec une solution d'urée à l'aide d'un pulvérisateur équipé d'une rampe d'aspersion de $6 \mathrm{~m}$ de long. La paille aspergée est immédiatement bottelée à l'aide d'une ramasseuse-presse, mise en meules et couverte hermétiquement avec des feuilles en plastique noir. Le nombre de bottes ainsi traitées a été de 400 .

\subsection{Traitement de la paille à l'urée sur bottes (paille PTUB)}

400 bottes de la même paille que celle traitée sur andain ont été rangées en meule. Chaque couche de bottes est aspergée avec la même solution d'urée que dans l'aspersion sur andain, à l'aide d'arrosoirs manuels. La meule est ensuite couverte avec des feuilles en plastique noir, de façon hermétique. 


\subsection{Conditions de traitement}

Pour chaque traitement, la quantité de paille traitée a été de 7,2 tonnes. Le traitement à l'urée a été effectué avec $6 \mathrm{~kg}$ d'urée dans $40 \mathrm{~kg}$ d'eau pour $100 \mathrm{~kg}$ de MS de paille. La température ambiante moyenne durant le traitement a été de $31{ }^{\circ} \mathrm{C}$. La durée de confinement a été de 2 mois, du 23 juillet au 13 septembre 1993.

\subsection{Essai 1 : Mesure de la digestibilité de la paille}

La digestibilité in vivo a été mesurée sur cinq moutons non castrés, de race locale OuledDjellal, pesant $57 \mathrm{~kg}$, placés dans des cages à métabolisme, au cours de trois périodes successives de $21 \mathrm{j}$ séparées par une période de transition de $7 \mathrm{j}$. Il s'agit de la même méthodologie de mesure que celle pratiquée à l'Inra de Theix [5]. Les animaux ont reçu successivement la paille non traitée (PNT), la paille traitée sur andain au champ (PTUA) et la paille traitée en bottes (PTUB).

Les animaux ont reçu en outre, une complémentation en concentré $(\mathrm{Cl}=83 \%$ d'orge et $17 \%$ de gros son). Avant l'essai, ils ont été traités contre les parasites internes et externes.

La paille a été distribuée à volonté (10 à $15 \%$ de refus) en deux repas par jour alors que le concentré a été offert à raison de 260 $\mathrm{g} / \mathrm{j} / \mathrm{animal}$ en un seul repas.

La digestibilité de la paille a été calculée à partir de celle de la ration entière. Pour cela, la digestibilité du concentré a été calculée à partir des tables Inra [22] pour la matière organique (MO) et les matières azotées (MAT) et déterminée par une méthode in vitro [29] pour la cellulose brute (CB).

\subsection{Essai 2 : Croissance des agneaux}

Trente agneaux de race Ouled Djellal, choisis dans un troupeau de cent têtes en fonction de l'âge (110 à $115 \mathrm{j})$ et du poids vif ont été répartis au hasard en trois lots de dix animaux chacun et de poids vifs comparables (lot $1=20,7 \pm 1,8 ; \operatorname{lot} 2=20,7 \pm 2,1 ;$ lot $3=20,6 \pm 1,9 \mathrm{~kg}$ ). Avant l'essai, les animaux ont été traités contre les parasites internes et externes.

Le lot 1 a reçu de la paille non traitée alors que les lots 2 et 3 ont reçu respectivement de la paille traitée à l'urée sur andain et sur bottes. En outre, le lot 1 a reçu $570 \mathrm{~g} / \mathrm{j} / \mathrm{animal}$ d'un concentré $\mathrm{C} 2$ et les autres lots $520 \mathrm{~g} / \mathrm{j} / \mathrm{animal}$ d'un concentré C3 dont les caractéristiques sont données dans le tableau I.

La paille a été offerte ad libitum ( 10 à $15 \%$ de refus) en deux repas par jour à 10 et 16 heures. Les concentrés C2 et C3 ont été distribués en deux repas par jour à 9 et 14 heures.

Les aliments distribués et refusés ont été pesés chaque jour. Les animaux ont été pesés tous les $20 \mathrm{j}$, à jeun et individuellement.

La durée de l'essai a été de $121 \mathrm{j}$ dont $21 \mathrm{j}$ d'adaptation des animaux aux régimes alimentaires.

\subsection{Analyses chimiques et calculs statistiques}

Les teneurs en matière sèche, en cellulose brute, en matières azotées totales et en cendres ont été déterminées selon les méthodes de l'AOAC [3].

Une analyse de variance a été effectuée par le test de Fischer et la comparaison des moyennes par le test de Newman-Keuls.

Tableau I. Composition centésimale des concentrés.

\begin{tabular}{ccccccc}
\hline Aliments & Orge & Gros son & Tourteau soja & CMV & Calcaire & Sel \\
\hline C1 & 83 & 17 & - & - & - & - \\
C2 & 54,48 & 21,03 & 18,77 & 1,72 & 2,51 & 1,40 \\
C3 & 22,14 & 72,23 & - & 1,72 & 2,51 & 1,40 \\
\hline
\end{tabular}

$\mathrm{C} 1, \mathrm{C} 2$ et $\mathrm{C} 3$, concentrés 1,2 et $3 ; \mathrm{CMV}$, complément minéral vitaminé. 


\section{RÉSULTATS}

\subsection{Effet des traitements sur la composition chimique de la paille}

La quantité d'eau ajoutée étant de 40 $\mathrm{kg}$ par $100 \mathrm{~kg}$ de MS de paille (tableau II), la teneur en MS des pailles traitées a été de $77,3 \%$ avec la paille PTUA et de $76,8 \%$ avec la paille PTUB, contre $91,6 \%$ pour la paille PNT.

Le traitement à l'urée a diminué la teneur en $C B$ de la paille de 2,3 points (PTUA) et de 3,0 points (PTUB) par rapport à la paille PNT (tableau II).

Les teneurs en MAT ont été augmentées de 3,8 points avec la paille PTUA et de 7,5 points avec la paille PTUB, soit des taux de fixation d'azote respectifs de 22,0 et $42,9 \%$ (tableau II).

\subsection{Résultats observés avec les moutons}

\subsubsection{Effet des traitements sur l'ingestion des pailles}

Les pailles traitées ont été mieux ingérées par les moutons que la paille non traitée et cela indépendamment du type de traitement, les améliorations enregistrées sont de $34 \%$ avec la paille PTUA et de $39 \%$ avec la paille PTUB sans que les différences soient significatives entre les pailles traitées (tableau III).

\subsubsection{Effet des traitements sur la digestibilité des pailles}

Le traitement à l'urée améliore la digestibilité de la matière organique (dMO) de 9,7 points quelle que soit la technique utilisée (tableau III).

Les augmentations de la digestibilité de la cellulose brute (dCB) sont comparables entre la paille PTUB et la paille PTUA avec respectivement 18,3 et 19,1 points (tableau III).

Les augmentations de la digestibilité des matières azotées totales (dMAT) enregistrées avec les pailles PTUA $(32,1$ points) et PTUB $(33,4$ points) ont été comparables (tableau III).

\subsection{Résultats observés avec les agneaux}

\subsubsection{Ingestion des rations}

Les rations à base de paille non traitée ou traitée à l'urée par aspersion mécanique sur

Tableau II. Composition chimique des concentrés et des pailles.

\begin{tabular}{|c|c|c|c|c|c|}
\hline & \multirow[b]{2}{*}{ MS (\%) } & \multicolumn{4}{|c|}{ Composition chimique en $\%$ de MS } \\
\hline & & $\mathrm{MO}$ & MAT & $\mathrm{CB}$ & $\mathrm{Nf} / \mathrm{Na}(\%)$ \\
\hline $\mathrm{Cl}$ & 88,7 & 96,6 & 12,6 & 7,8 & \\
\hline $\mathrm{C} 2$ & 84,5 & $89,5 \pm 1,7^{\mathrm{a}}$ & $17,1 \pm 1,8^{\mathrm{a}}$ & $4,9 \pm 0,1^{a}$ & \\
\hline $\mathrm{C} 3$ & 85,7 & $89,1 \pm 1,9^{\mathbf{a}}$ & $11,9 \pm 0,6^{\mathrm{b}}$ & $7,9 \pm 0,8^{b}$ & \\
\hline PNT & $91,6 \pm 2,1$ & $94,4 \pm 0,9$ & $3,3 \pm 1,7$ & $39,1 \pm 1,7$ & \\
\hline PTUA & $77,3 \pm 7,2$ & $93,5 \pm 0,3$ & $7,1 \pm 0,6$ & $36,8 \pm 1,4$ & 22,0 \\
\hline PTUB & $76,8 \pm 6,5$ & $93,8 \pm 0,6$ & $10,9 \pm 0,4$ & $36,1 \pm 1,2$ & 42,9 \\
\hline
\end{tabular}

PTUA, paille traitée à l'urée par aspersion mécanique sur andain ; PTUB, paille traitée à l'uréc par aspersion manuelle sur bottes : PNT, paille non traitée ; $\mathrm{C} 1$, concentré $1 ; \mathrm{C} 2$, concentré $2 ; \mathrm{C} 3$, concentré 3 ; MS, matière sèche ; $\mathrm{MO}$, matière organique ; $\mathrm{CB}$, cellulose brute ; $\mathrm{Nf}$, azote fixé sur la paille ; $\mathrm{Na}$, azote appliqué ; sur une même colonne, les valeurs marquées d'un même signe sont comparables au seuil de $5 \%$. 
Tableau III. Ingestibilité et digestibilité des pailles mesurées sur moutons.

\begin{tabular}{lccc}
\hline Régimes alimentaires & PNT + Cl & PTUA + C1 & PTUB + C1 \\
\hline $\begin{array}{l}\text { Ingestibilité (gMS/kg P0.75) } \\
\quad \text { Ration }\end{array}$ & $46,7 \pm 2,0^{\mathrm{a}}$ & $58,0 \pm 2,9^{\mathrm{b}}$ & $59,4 \pm 1,1^{\mathrm{b}}$ \\
$\quad$ Paille seule & $32,8 \pm 5,5^{\mathrm{a}}$ & $44,0 \pm 2,9^{\mathrm{b}}$ & $45,7 \pm 0,8^{\mathrm{b}}$ \\
$\begin{array}{l}\text { Digestibilité (\%) } \\
\quad \text { dMO de la ration }\end{array}$ & $58,7 \pm 5,0^{\mathrm{a}}$ & $65,1 \pm 2,5^{\mathrm{c}}$ & $63,9 \pm 1,6^{\mathrm{b}}$ \\
$\quad \begin{array}{l}\text { Paille seule } \\
\quad \text { dMO }\end{array}$ & $46,3 \pm 2,8^{\mathrm{a}}$ & $56,0 \pm 4,3^{\mathrm{b}}$ & $56,0 \pm 0,7^{\mathrm{b}}$ \\
$\quad$ dMAT & $24,8 \pm 0,8^{\mathrm{a}}$ & $56,9 \pm 2, \mathrm{1}^{\mathrm{b}}$ & $58,2 \pm 2,0^{\mathrm{b}}$ \\
$\quad$ dCB & $46,8 \pm 7,8^{\mathrm{a}}$ & $65,9 \pm 1,5^{\mathrm{b}}$ & $65,1 \pm 0,5^{\mathrm{b}}$ \\
MAND (g/kg de MS & 25 & 31 & 45 \\
\hline
\end{tabular}

PTUA, paille traitée à l'urée par aspersion mécanique sur andain ; PTUB, paille traitée à l'urée par aspersion manuelle sur bottes; $\mathrm{PNT}$, paille non traitée ; $\mathrm{Cl}$, concentré 1 ; dMO, digestibilité de la matière organique ; dMAT, digestibilité des matières azotées totales ; $\mathrm{dCB}$, digestibilité de la cellulose brute ; MAND, matières azotées non digestibles ; sur une même ligne, les valeurs portant un même signe, sont comparables au seuil de $5 \%$.

andain ou manuelle sur bottes ont été ingérées en quantités comparables (tableau $I V$ ).

\subsubsection{Ingestion de la paille seule par les agneaux}

L'ingestion de la paille PTUA par les agneaux $\left(38,2 \mathrm{gMS} / \mathrm{kgP}^{0.75}\right)$ a été identique à celle de la paille PTUB $(38,7$ $\left.\mathrm{gMS} / \mathrm{kgP}^{0.75}\right)$. Par rapport à la paille PNT ingérée à environ $32 \mathrm{gMS} / \mathrm{kgP}^{0.75}$, les améliorations de l'ingestion enregistrées sont de 15,6 à $16,4 \%$ avec la paille traitée (tableau $I V$ ).

\subsubsection{Gains de poids vif moyens quotidiens (GMQ) et indice de consommation}

Le meilleur GMQ (112 g) a été observé pour la paille traitée par aspersion mécanique sur andain au champ (PTUA + C3) ; il est de $103 \mathrm{~g}$ avec la paille traitée par aspersion manuelle sur bottes (PTUB + C3), ce dernier n'étant pas significativement différent de celui réalisé avec le régime $\mathrm{PNT}+\mathrm{C} 2$ (103 contre $104 \mathrm{~g}$; tableau $(V)$.
Les différences observées dans les GMQ n'ont pas entraîné de différence significative entre les indices de consommation des différents régimes. Ces indices ont varié entre 9,2 et 9,9 kg de MS/ $\mathrm{kg}$ de gain de poids vif. Les coûts alimentaires du $\mathrm{kg}$ de gain de poids vif ont varié entre 44,0 D.A (PTUA + C3) et 50,0 D.A $(\mathrm{PNT}+\mathrm{C} 2)$. Ainsi, le coût alimentaire du $\mathrm{kg}$ de gain de poids vif a été surtout déterminé par le prix des aliments utilisés dans chaque régime (tableau IV).

\section{DISCUSSION}

La teneur en MS de la paille traitée par aspersion manuelle sur bottes (PTUB) devrait être inférieure à celle de la paille traitée par aspersion mécanique sur des andains hétérogènes (PTUA) en raison des pertes de solution d'urée. Or il n'en est rien ; ces pertes seraient alors compensées par l'absence d'évaporation lors de l'aspersion mécanique réalisée le matin au lever du jour et sur une paille vraisemblablement un peu plus humide que plus tard dans la journée. 
Tableau IV. Caractéristiques des rations ingérées, indices de consommation et performances des agneaux.

\begin{tabular}{lccc}
\hline Lots & 1 (PNT + C2) & 2(PTUA + C3) & 3 (PTUB + C3) \\
\hline Nombre d'animaux & 10 & 10 & 10 \\
Durée de l'essai (j) & 100 & 100 & 100 \\
$\begin{array}{l}\text { Ingestibilité (gMS/kg P0.75) } \\
\quad \text { Ration }\end{array}$ & $82,5^{\mathrm{a}}$ & & \\
$\quad$ Paille seule & $32,3^{\mathrm{a}}$ & $32,4^{\mathrm{a}}$ & $85,1^{\mathrm{a}}$ \\
Paille dans la ration (\%) & 39 & 46 & $38,7^{\mathrm{b}}$ \\
MAT dans la ration (\%) & 11,7 & 9,7 & 45 \\
NNP/Nt de la ration (\%) & & 18 & 11,4 \\
$\begin{array}{l}\text { Poids vif initial (kg) } \\
\text { Poids vif final (kg) }\end{array}$ & 20,7 & 20,7 & 30 \\
Gain moyen quotidien (g) & $104,0 \pm 6,0^{\mathrm{b}}$ & $112,0 \pm 2,1^{\mathrm{b}}$ & $103,0 \pm 3,6^{\mathrm{b}}$ \\
$\begin{array}{l}\text { Indice de consommation } \\
\text { (kg MS/kg de gain de PV) }\end{array}$ & $9,5 \pm 0,5^{\mathrm{a}}$ & $9,8 \pm 0,1^{\mathrm{a}}$ & $9,9 \pm 0,4^{\mathrm{a}}$ \\
$\begin{array}{l}\text { Coût alimentaire du kg } \\
\text { de gain de PV (D.A.) }\end{array}$ & 50 & 44 & 46 \\
\hline
\end{tabular}

PTUA, paille traitée à l'urée par aspersion mécanique sur andain ; PTUB, paille traitée à l'urée par aspersion manuelle sur bottes ; PNT, paille non traitée; $\mathrm{C} 2$, concentré $2 ; \mathrm{C} 3$, concentré 3 ; MAT, matières azotées totales ; $\mathrm{NNP}$, azote non protéique ; $\mathrm{Nt}$, azote total ; PV, poids vif ; D.A, dinar algérien ; sur une même ligne, les valeurs portant le même signe sont comparables au seuil de $5 \%$.

Les traitements (PTUA et PTUB) ont diminué la teneur en cellulose brute de la paille. Une baisse plus faible $(0,6$ à 1,1 points) a été enregistrée par Houmani [21] sur paille de blé traitée avec $50 \mathrm{~g}$ d'urée dans $400 \mathrm{~g}$ d'eau par $\mathrm{kg}$ de MS. Messaoudi et Bendhia [25] ont observé une diminution de 2,2 points avec une paille d'orge traitée avec $50 \mathrm{~g}$ d'urée dans $250 \mathrm{~g}$ d'eau par $\mathrm{kg}$ de MS. Selon Cloete et Kritzinger [13], cette baisse de la teneur en CB des pailles traitées serait due à une solubilisation partielle des hémicelluloses par le traitement à l'urée. L'analyse chimique des parois cellulaires des pailles [30] fournirait certainement une meilleure explication puisque la $\mathrm{CB}$ contient en général, très peu d'hémicelluloses.
Les taux de fixation d'azote sont largement inférieurs à ceux rapportés par Gupta et al. [17] puis Abdouli et al. [1] avec 89 et $79 \%$ pour des pailles traitées avec $40 \mathrm{~g}$ d'urée dans respectivement 500 et $450 \mathrm{~g} \mathrm{~d}$ 'eau par $\mathrm{kg}$ de MS. Le taux de fixation d'azote réalisé avec l'aspersion mécanique sur andain est faible à cause des pertes de solution d'urée dues aux conditions de traitement (quantité de paille hétérogène sur andain, forme irrégulière de l'andain et débit constant de la rampe d'aspersion). Ces observations sont également rapportées par Chenost [8]. Chenost et Besle [9] notent des taux de fixation d'azote de l'ordre de $25 \%$ pour une paille de blé traitée avec $6 \%$ d'urée avec 25 à $30 \%$ d'humidité, à l'aide d'une rampe 
d'aspersion fixée au-dessus du 'pick up' de la presse, ce qui réduirait les pertes de solution d'urée lors de l'aspersion. Le passage d'un rateau-faneur avant l'aspersion mécanique pourrait limiter les pertes de solution d'urée par la mise en forme unique des andains.

Les augmentations d'ingestion des pailles traitées (34 et $39 \%$ ) confirment les résultats de Borhami et al. [6], Alibes et al. [2] et Chenost et Besle [9]. Ces améliorations sont comparables à celle observée par Munoz [26] avec une paille de blé traitée à l'urée complémentée avec divers sous-produits, avec une augmentation de $39 \%$ (33 à $46 \mathrm{~g} \mathrm{MS} / \mathrm{kg} \mathrm{P}^{0.75}$ ).

L'amélioration de la dMO notée pour les pailles traitées $(9,7$ points) est inférieure à celles observées par Abdouli et al. [1] avec 14,8 points sur une paille traitée à $4 \%$ d'urée complémentée avec $200 \mathrm{~g}$ d'un mélange à $94 \%$ d'orge et $6 \%$ de CMV et par Saleh et al. [28] avec 12,4 points avec une paille traitée à l'urée à $200 \mathrm{~g}$ par litre complémentée avec $70 \mathrm{~g}$ de tourteau de soja et $20 \mathrm{~g}$ de CMV.

L'action alcaline du traitement à l'urée $[4,14]$ expliquerait l'amélioration de la $\mathrm{dCB}$ avec les pailles traitées et serait donc identique entre le traitement par aspersion mécanique sur andain au champ et le traitement par aspersion manuelle sur bottes.

Les augmentations de la dMAT des pailles traitées sont inférieures à celle notée par Cloete et Kritzinger [12] sur paille traitée à l'urée avec 53.2 points $(-3.3$ à $49.5 \%$ ) et se rapprochent de celle observée par Chermiti et al. [11] avec une paille traitée à l'urée avec 38,2 points $(21,1$ à $59,4 \%)$. Avec les pailles traitées, la différence de 3,7 points dans la teneur en MAT n'a pas entraîné de digestibilité (dMAT) différente. Les teneurs en matières azotées non digestibles (MAND) ont donc été plus élevées pour la paille PTUB avec $45 \mathrm{~g} / \mathrm{kg}$ MS par rapport à la paille PTUA avec $31 \mathrm{~g} / \mathrm{kg}$ MS et à la paille PNT avec $25 \mathrm{~g} / \mathrm{kg}$ MS. On sait que même s'il augmente la digestibilité des MAT, le traitement à l'ammoniac (anhydre ou via urée) augmente l'excrétion fécale azotée par suite d'une utilisation de l'azote fixé par les micro-organismes du rumen moins bonne que prévue [19]. Toutefois, l'augmentation des MAND plus importantes avec la paille PTUB qu'avec la paille PTUA permet de se demander si l'urée n'a pas été moins bien transformée en ammoniac avec le traitement en bottes (PTUB) qu'avec le traitement en andain (PTUA). Ceci expliquerait le taux de «fixation » artificiellement plus élevé observé (part de l'urée dans les MAT plus importante) et l'augmentation modeste de la dMO et de la quantité ingérée pour la paille PTUB, pratiquement identique à celle de la paille PTUA, moins riche en MAT parce que traitée avec moins d'urée par suite des pertes lors de l'aspersion des andains.

L'enrichissement de la paille en azote par le traitement à l'urée stimule la prolifération de la microflore ruminale et son activité cellulolytique [7]. La paille est dégradée plus rapidement et transite plus vite. Les quantités qui sont volontairement ingérées sont donc plus importantes (régulation physique de l'appétit). Selon Males et Gaskins [24] et Abdouli et al. [1], l'augmentation du taux d'humidité avec les pailles traitées est également responsable de l'amélioration de l'ingestion de la paille traitée à l'urée. Par ailleurs, les améliorations de la quantité de paille traitée ingérée par les agneaux sont inférieures à celles observées avec les moutons adultes lors du test de digestibilité (amélioration de 34 à $39 \%$ ) en raison d'une distribution de concentré plus élevée aux agneaux.

Les différents régimes distribués aux agneaux permettent des gains moyens quotidiens peu différents mais avec moins de concentré pour les deux régimes à base de pailles traitées que le régime avec la paille non traitée. 


\section{CONCLUSION}

La technique par aspersion mécanique de l'urée en solution sur andain au champ pour le traitement de la paille de blé est aussi intéressante que la technique par aspersion manuelle sur bottes.

À l'exception de la teneur en MAT qui est légèrement inférieure avec la paille traitée par aspersion mécanique sur andain, les augmentations de l'ingestibilité et de la digestibilité sont comparables à celles permises par l'aspersion manuelle sur bottes et les performances des agneaux sont pratiquement identiques.

Les indices de consommation sont comparables entre les régimes avec paille traitée et paille non traitée mais se traduisent néanmoins, par une économie sur le coût alimentaire de 8 à $12 \%$ par kg de croît par rapport au régime témoin à base de paille non traitée.

On peut donc traiter au champ ou à la ferme selon la facilité du travail. Cependant au champ, il y a lieu de faire attention aux pertes d'urée. Pour cela, des travaux complémentaires sont nécessaires pour optimiser l'incorporation et l'homogénéisation de la solution d'urée au sein de la masse de paille en quantité hétérogène sur l'andain au champ par le passage, par exemple, d'un râteau faneur avant le traitement pour une mise en forme unique des andains.

\section{RÉFÉRENCES}

11] Abdouli H., Khorchani K., Kraiem K., Traitement de la paille à l'urée. II. Effets sur la croissance des taurillons et sur la digestibilité, Rev. Four. 114 (1988) 167-176.

[2] Alibes K., Munoz. F., Faci F., Anhydrous ammonia-treated cereal straw for animal feeding: some results from the mediterranean area. Anim. Feed Sci. Technol. 10 (1984) 239-246.

[3] Aoac, Official methods of analysis, 12th edn. Washington, DC, 1975.
14] Bacon S.J.D., Chesson A., Gordon A.H., Deacitylation and enhancement of digestibility, Agric. Environm. 6 (1981) 115-125.

[5] Benahmed H., Dulphy J.P., Note sur la valeur azotée des fourrages pauvres traités par l'urée ou l'ammoniac, Ann. Zootech. 34 (1985) 335-346,

[6] Borhami B.E.A., Sundstol F., Garo T.H., Studies on ammonia-treated straw. II. Fixation of ammonia in treated straw by spraying with acids, Anim. Feed Sci. Technol. 7 (1982) 53-59.

[7] Chenost M., Utilisation digestive des pailles, Options méditerranéennes Série A. Sém. Méd. 16 (1991) 67-72.

[8] Chenost M., Les facteurs de réussite du traitement des pailles à l'urée. Opt. Méd. Série B 6 ( l 994) 47-60.

[9] Chenost M., Besle J.M., Les pailles traitées à l'ammoniac provenant de l'hydrolyse de l'urée dans l'alimentation des génisses de race laitière en croissance hivernale, Ann. Zootech. 41 (1992) 153-167.

[10] Chermiti A., Nefzaouj A., Cordesse R., Paramètres d'uréolyse et digestibilité de la paille traitéc à l'urée, Ann. Zootech. 38 (1989) 63-72.

[11] Chermiti A., Nefzaoui A., Teller E., Vanabelle M., Optimisation du traitement des pailles de céréales à l'ammoniac et à l'urée. 1 . Evaluation de l'efficacité du traitement à partir des pertes de produits volatils, Rev. de l'Agriculture Landbouwtijdschrift 44 (1991) 973-982.

112] Clocte S.W.P., Kritzinger N.M., Urea ammoniation compared to urea supplementation as method of improving the nutritive value of wheat straw for sheep, S. Afric. J. Anim. Sci. 4 (1984) 59-63.

[13] Cloete S.W.P., Kritzinger N.M.. A laboratory assessment of various treatment conditions affecting the ammoniation of wheat straw by urea. 1: The effect of temperature. moisture level and treatment period, S. Afric J. Anim. Sci. 14 (1985) 55-58.

114] Cordesse R., Technologie de traitement des pailles à l'ammoniac, in: Les fourrages secs, récolte traitement, utilisation, Inra, Paris 1987. pp. 231-242.

[15] Dias Da Silva A., Sundstol F., Urea as a source of ammonia for improving the nutritive value of wheat straw, Anim. Feed. Sci. Technol. 14 (1986) 67.

[16] Guessous F., Rihani N., Utilisation des pailles dans l'alimentation des ruminants dans les pays de la zone méditerranéenne. $2^{e}$ rapport annuel du projet STD pailles, contrat de recherche no TS 2A-0250-M(CD). 1991. 
[17] Gupta R., Singh A.P., Gupta B.S., Strivastava J.P., Maheshawri P.K., Effet of ammonia (generated from urea) on proximate constituents of paddy straw, Ind. J. Anim. Health 24 (1985) 101.

[18] Hadjipanayiotou M., The effect of ammoniation using urea on the intake and nutritive value of chopped barley straw, Grass For. Sci. 37 (1982) 89-93.

[19| Hassen L., Chenost M., Tentative explanation of the high abnormal faecal nitrogen excretion with poor quality roughages-treated with ammonia, Anim. Feed Sci. Technol. 38 (1992) 25-34.

[20] Houmani M., Evaluation du bilan fourrager en Algérie, in : $2^{\mathrm{ew}}$ Jounées d'étude sur la production et la technologic du lait, 13-14 mar 1996. Institut National de Formation Supćrieure en Agronomic. Mostaganem, 1996.

121 Houmani M.. Influence de la couverture des meules dans le traitement des pailles à l'urće. Fourrages 150)(1997) 235-243.

[22] Inra, Alimentation des bovins, ovins et caprins, Inra, Paris, France, 1988.

1231 Lawrence A., Yakhlef H., Triki S., Chabaka R., $2^{\mathrm{C}}$ rapport annuel du projet $\mathrm{STD}$ pailles, contrat de recherche no. TS $2 \mathrm{~A}-0250-\mathrm{M}(\mathrm{CD})$. 1991.

24] Males J.R., Gaskins L.T., Growth nitrogen. dry matter digestibility and ruminal characteristics associated with ammoniated straw diets, J. Anim. Sci. 55 (1982) 505.
[25] Messaoudi L., Bendhia M., La paille traitée à l'urée couverte avec de la terre comparée à celle traitée à l'urée et à l'ammoniac, couverte avec des feuilles en plastique, in «Rapport d'activité 1994 », Réseau régional de vulgarisation pour l'amélioration des pailles et des résidus de cultures lignifiés dans laalimentation des ruminants, GCP/INT/523/FRA, Alexandrie, 12-15 nov, 1994.

[26] Munoz F., Study of the influence of temperature on the effect of treatment of wheat straw with urea in an aqueous solution, $2^{\mathrm{e}}$ rapport annuel de projet STD paille. contrat de recherche, TS 2A-0250-M (CD), 1991

[27] Sahnoune S., Girard L., Besle J.M., Chenost M., A kinetic study of ammoniation of straw either via the hydrolysis of urea or by anhydrous ammonia, $\mathrm{VI}^{\mathrm{es}}$ Journées de recherche sur l'alimentation et la nutrition des herbivores. INA P-G, 21-22 mars, 1990

[28] Saleh H., Koenig M., Millot J.C., Tisserand J.L., Étude comparative du traitement par une solution de soude ou par une solution d'uréc sur l'ingestibilité et la digestibilité d'une paille. $V$ “ journées de recherche sur l'alimentation et la nutrition des herbivores, INA P-G, 16-17 mars, 1989.

[29] Tilley J.M.A., Terry R.A., A two-stage technique for the in vitro digestion of forage crops, J. Br. Grassl. Soc. 18 (1963) 104-111.

130| Van Soest P.J., Wine R.H., Use of detergents in the analysis of fibrous feeds. IV. Determination of plant cell wall constituants, J. Assoc. Off. Agric. Chem. 50 (1967) 50-55. 